\title{
A Stepped Wedge Cluster-Randomized Trial Assessing the Impact of a Riverbank Filtration Intervention to Improve Access to Safe Water on Health in Rural India
}

\author{
Sarah L. McGuinness, ${ }^{1 \star}$ Joanne O'Toole, ${ }^{1}$ Andrew B. Forbes, ${ }^{1}$ Thomas B. Boving, ${ }^{2,3}$ Kavita Patil, ${ }^{4}$ Fraddry D'Souza, ${ }^{4}$ \\ Chetan A. Gaonkar, ${ }^{4}$ Asha Giriyan, ${ }^{4}$ S. Fiona Barker, ${ }^{1}$ Allen C. Cheng, ${ }^{1}$ Martha Sinclair, ${ }^{1}$ and Karin Leder ${ }^{1}$ \\ ${ }^{1}$ School of Public Health and Preventive Medicine, Monash University, Melbourne, Australia; ${ }^{2}$ Department of Geosciences, University of Rhode \\ Island, Kingston, Rhode Island; ${ }^{3}$ Department of Civil and Environmental Engineering, University of Rhode Island, Kingston, Rhode Island; ${ }^{4}$ The \\ Energy and Resources Institute (TERI), Goa, India
}

\begin{abstract}
Sustainable and low-cost methods for delivery of safe drinking water in resource-limited settings remain suboptimal, which contributes to global diarrhea morbidity. We aimed to assess whether delivery of riverbank filtrationtreated water to newly installed water storage tanks (improved quality and access, intervention condition) reduced reported diarrhea in comparison to delivery of unfiltered river water (improved access alone, control condition) in rural Indian villages. We used a stepped wedge cluster-randomized trial (SW-CRT) design involving four clusters (villages). Selection criteria included village size, proximity to a river, and lack of existing or planned community-level safe water sources. All adults and children were eligible for enrollment. All villages started in the control condition and were sequentially randomized to receive the intervention at 3-month intervals. Our primary outcome was 7-day-period prevalence of self- or caregiver-reported diarrhea, measured at 3-month intervals (five time points). Analysis was by intention to treat. Because blinding was not possible, we incorporated questions about symptoms unrelated to water consumption to check response validity (negative control symptoms). We measured outcomes in 2,222 households (9,836 participants). We did not find a measurable reduction in diarrhea post-intervention (RR: 0.98 [95\% Cl: 0.24-4.09]); possible explanations include low intervention uptake, availability of other safe water sources, low baseline diarrheal prevalence, and reporting fatigue. Our study highlights both the difficulties in evaluating the impact of real-world interventions and the potential for an optimized SW-CRT design to address budgetary, funding, and logistical constraints inherent in such evaluations.
\end{abstract}

\section{INTRODUCTION}

Diarrhea remains among the leading causes of death in young children worldwide and disproportionately affects vulnerable populations in low- and middle-income countries (LMICs). ${ }^{1,2}$ Diarrhea transmission can occur through multiple pathways, including through consumption of water contaminated with feces. ${ }^{3}$ The Sustainable Development Goal (SDG) to ensure universal and equitable access to "safely managed drinking water" by 2030 (water that is free from fecal and chemical contamination, located on premises, and available when needed) $)^{4,5}$ recognizes the need to improve both water quality and access, particularly in LMICs. This ambitious target reflects the understanding that ready access to safe water is essential not only to prevent diarrhea but also to facilitate personal hygiene and support socioeconomic development. ${ }^{6-8}$ Global progress has been made toward this SDG, but in 2015, an estimated 844 million people worldwide still lacked even a basic drinking water service and 2.1 billion people lacked safely managed drinking water. ${ }^{4}$ Fecal contamination of the environment and of drinking water supplies is widespread in LMICs and affects all water source types, including piped supplies. ${ }^{9}$

India alone accounts for approximately $20 \%$ of the global burden of childhood deaths due to diarrhea, with an estimated 105,000 children aged less than 5 years dying from diarrhea every year. ${ }^{10}$ With a population of more than 1.3 billion people, India faces significant challenges in meeting the growing water supply needs of its population. ${ }^{11}$ There are disparities in water access, with $56 \%$ of the top $20 \%$ household income group having access to piped water, compared with only $6 \%$

* Address correspondence to Sarah L. McGuinness, Monash University, SPHPM 553 St. Kilda Rd., Melbourne 3004, Australia. E-mail: sarah.mcguinness@monash.edu of those in the bottom $20 \% .{ }^{12}$ An estimated 13 million people living in rural India continue to rely on untreated surface water, such as river water, for drinking. ${ }^{4}$ However, pollution related to agricultural runoff, open defecation, and inadequate upstream treatment of domestic sewage and industrial wastewater make untreated river water an unsuitable drinking water source. ${ }^{11}$

Water interventions in LMICs can be delivered at a household level (e.g., point-of-use chlorination, filtration, or solar disinfection), a community level (e.g., improved water sources such as protected groundwater, harvested rainwater, or filtered water), or a municipal level. ${ }^{13}$ Most previous studies in LMICs have evaluated household-level point-of-use interventions, which are logistically easier to study and randomize. While available evidence suggests that point-of-use interventions can lead to reductions in diarrheal disease, substantial heterogeneity has been observed in effect sizes between individual studies, ${ }^{13-16}$ with larger effects typically seen in trials with higher adherence. ${ }^{13}$ However, much of this evidence is based on efficacy trials with intensive follow-up, and few studies have formally evaluated long-term adoption and sustainability, ${ }^{17}$ resulting in questions regarding the acceptability and scalability of these interventions. ${ }^{6,13,14,18,19}$ Furthermore, recent high-quality studies of point-of-use water treatment interventions with longer follow-up periods (2 years) have failed to demonstrate reductions in diarrheal disease despite high levels of uptake. ${ }^{20-22}$ As point-of-use interventions do little to address issues with water access or availability, they are typically viewed as an interim solution until homes can be reached with safely managed, piped water connections. ${ }^{6,13}$ Community-level source-based interventions can potentially address both water quality and access, and offer a more sustainable, long-term approach, although few trials have quantified improvements in water access by measuring water usage at the household level. ${ }^{8}$ However, 
these interventions are expensive and logistically more challenging to implement and study; cost and logistical constraints need to be balanced against maximizing statistical power to detect a difference in diarrheal disease.

Riverbank filtration is an inexpensive, source-based, water quality improvement technique that uses natural, autoregenerative filtration processes to improve water quality. ${ }^{23}$ Riverbank filtration systems consist of one or more wells, electric submersible pump(s), and pipeline through which water can be delivered to water storage tanks, or directly to homes. As the name suggests, riverbank filtration systems are designed to treat river water and are a potential water treatment solution for communities adjacent to polluted rivers. Although the design and installation of riverbank filtration systems requires engineering expertise, once in place, local communities can be trained to operate and maintain them long term, although this may require some dedicated resourcing.

We set out to evaluate the effects of improving water quality and access versus improving water access alone on the health of adults and children in rural India using a stepped wedge cluster-randomized trial (SW-CRT) design. Specifically, we aimed to assess whether delivery of riverbank filtration-treated water to a series of newly installed water storage tanks (improved quality and access) reduced reported diarrhea in comparison to delivery of unfiltered river water (improved access alone).

\section{MATERIALS AND METHODS}

Study design. Details of our study design and methodology have been published previously. ${ }^{24}$ Briefly, we used a closed cohort SW-CRT design, ${ }^{25}$ with villages as the cluster unit. We conducted the study in four rural Indian villages (Figure 1). After obtaining written informed consent, we conducted household-level surveys to collect pretrial demographic and health data, and to provide pretrial hygiene education across all four villages (T0, February-May 2016). We then undertook a construction phase, involving installation of wells for riverbank filtration, and a new water distribution system consisting of polyvinyl chloride pipeline and a series of 2,000 to $3,000 \mathrm{~L}$ capacity water storage tanks with taps, distributed throughout all four villages (one tank per 25-30 households). Systems were operated by 5 to 7.5 horsepower pumps connected to the local electricity grid. After construction completion, the trial began with simultaneous continuous supply of untreated river water (control condition) to all villages, piped to water storage tanks using the new distribution systems (T1, October 2016). Riverbank filtration-treated water (intervention condition), piped to the same system of tanks, was then rolled out sequentially to each village in a random order at 12-week intervals (T2-T5). We conducted household-level surveys during each time period (T1-T5) of the trial. Surveys were completed within 6-8 weeks and were conducted simultaneously across all villages. Household-stored water samples were collected from a random sample of 160 households per village before each of the T1-T5 surveys; these results will be reported separately. Following conclusion of the SW-CRT (January 2018), we conducted qualitative focus groups with data collectors ("surveyors") and participants ("householders"); these results are reported separately. ${ }^{26}$

The study protocol was reviewed and approved by the Monash University Human Research Ethics Committee in Australia (CF/15/522 2015000248) and the Energy and Resources Institute Institutional Ethics Committee in India, and we registered the study with the Australian New Zealand Clinical Trials Registry (ACTRN12616001286437). We report our trial in line with the recently published Consolidated Standards of Reporting Trials (CONSORT) extension for the SW-CRT and include the CONSORT SW-CRT checklist as Supplemental Appendix $1 .^{27}$

Site selection. A detailed description of our site selection process has been published previously. ${ }^{28}$ We evaluated villages located within the upper Krishna watershed in the Athani Taluka of northwest Karnataka for inclusion. We selected villages based on several criteria, including size, community receptiveness, use of untreated river water as the main drinking water source, no imminent plans for a water supply

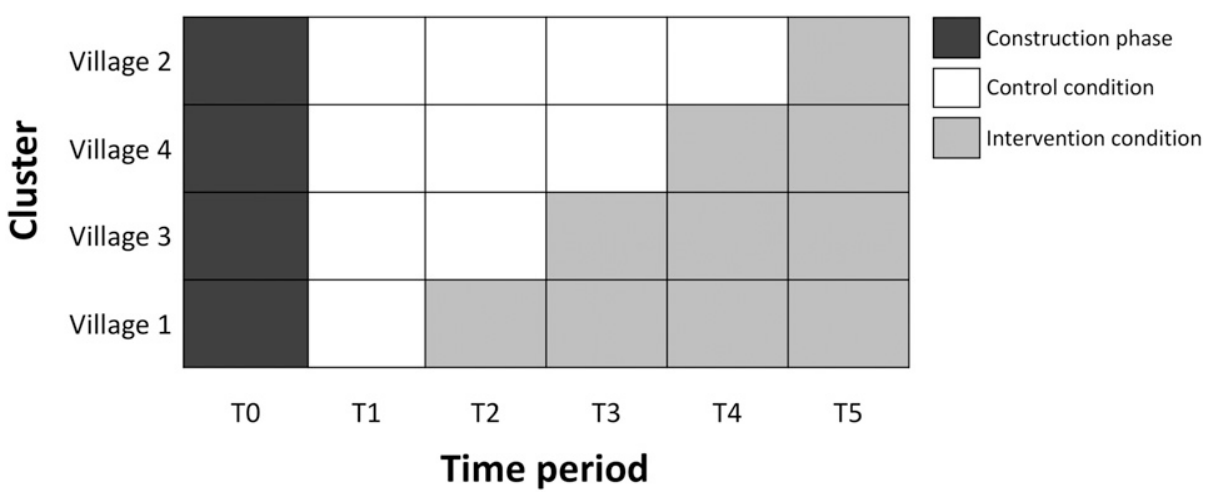

FIGURE 1. Stepped wedge schematic for the study. Each cell represents a time period where data were collected. Dark gray cells represent the pretrial time period (TO), during which we enrolled villages, provided pretrial hygiene education, and constructed water delivery systems. At the start of the trial (T1), we piped untreated river water to the newly installed water storage tanks (control condition, white cells) in all four villages. At each subsequent time point (T2-T5), one village was sequentially randomized to cross over to the intervention (riverbank filtration-treated water piped to water storage tanks, light gray cells) and remain in the intervention condition for the rest of the study. Village 1 was the first to receive the intervention at T2 (January 2017), followed by village 3 at T3 (April 2017), village 4 at T4 (July 2017), and village 2 at T5 (October 2017). Each period followed on immediately from the previous, but we allowed a transition period of 4 weeks after intervention implementation before beginning outcome measurements. 
upgrade, and availability of suitable land for riverbank filtration installation. We aimed to select geographically distinct villages to reduce the potential for contamination between clusters.

Study population. All households and individuals within each of the selected villages were eligible for enrollment. A self-nominated adult household representative provided household-level written informed consent for participation in household surveys, collection of household-stored water samples, and photography of household water storage containers and handwashing areas.

Randomization and blinding. Eligible villages were randomly assigned to one of four sequences (one village per sequence) for time of crossover from control to intervention. A coinvestigator (A. B. F.) performed randomization using a computer-generated list of random numbers and concealed the allocation sequence in sequentially numbered, opaque, sealed envelopes. The allocation sequence was revealed to other investigators one village at a time, with the next village to receive the intervention revealed a few weeks before intervention rollout. Blinding of participants to the intervention was not possible because of distinct visible components (connection of riverbank filtration wells to distribution systems and chlorination of the system before delivery) and potential differences in the taste and turbidity of untreated versus riverbank filtration-treated water. However, we did not overtly tell participants when the switch from control to intervention occurred. Survey questions relating to water use referred to "water storage tanks with the project logo" and did not specify the water type provided. Two investigators (A. B. F. and S. L. $M$.) performed independent, masked statistical analyses from raw datasets, with the order of allocation unmasked only after all analyses had been replicated.

Procedures. Following field surveys and site selection (July-August 2015), we used a participatory community model to gather information about existing water supply systems and plan the delivery of the riverbank filtration intervention. Google Earth images of each village and existing water supply plans (where available) were used to create a schematic of village water supply infrastructure, which was used by village leaders and study personnel to plan the location of wells, and distribution of water storage tanks and pipework. Following consultation with local landholders, Gram Panchayat (local government) leaders, and relevant parties, relevant approvals were sought at local, district, and state government levels. A single intervention delivery team sited and constructed wells for riverbank filtration in all villages, and prepared water delivery systems for intervention rollout.

Research assistants ("surveyors") fluent in the local language (Kannada) were recruited from local villages and consisted of 12 surveyor pairs (three per village), with equal gender representation where possible. Surveyors had a bachelor's degree (men) or a minimum of 10 years of formal schooling (women) and received a monthly stipend at appropriate local rates. Surveyors were responsible for enrollment, delivery of pretrial hygiene education to households, and collection of outcome data through household surveys. Surveyors received an initial week-long training session, followed by quarterly refresher sessions before each survey. Hygiene education was designed to promote handwashing and safe storage of water in the home and was centered around two open-source posters outlining key times for handwashing with soap and water, and safe storage of water in the home. ${ }^{29}$ Households were given an A5 laminated version of each poster, with advice to display these near the handwashing station and water storage containers, respectively. At subsequent visits, surveyors reinforced key education messages and checked for the presence of posters. Surveyors conducted surveys in pairs in the local language (Kannada), with a self-appointed adult household representative answering questions on behalf of other household members. Identifiable data were collected on a paper form, each household and individual was given a unique code, and coded demographic data were transcribed into an Excel spreadsheet. Survey responses were collected on handheld electronic tablet devices using household number and person number codes, with deidentified data transmitted to a central database via the cloud. Household-level questions predominated, but health outcome data were collected for all household members. Two field assistants with bachelor degrees in microbiology were recruited to collect and analyze river, well, water storage tank, and household water samples for microbial and chemical quality (findings reported separately). ${ }^{28}$

Outcomes. Based on the hypothesis that our intervention would reduce illness, our prespecified primary outcome was the 7-day-period prevalence of self- or caregiver-reported diarrhea, defined as three or more loose or watery stools within a 24-hour period. Secondary outcomes included the 7-dayperiod prevalence of acute respiratory infection (one or more of runny nose, sore throat, cough, or shortness of breath) and skin infection (one or more of localized redness, lump, swelling, or open sore/ulcer). We included self- or caregiverreported skin bruising/abrasion, urinary frequency, or headache as negative control symptoms to check response validity. ${ }^{30}$ Outcome data were collected through household surveys (T1-T5).

Sample size. Details of the sample size calculation have been published previously. ${ }^{24}$ Briefly, sample size was based on detecting a relative reduction in the 7-day-period prevalence of diarrhea, with $80 \%$ power and $\alpha=0.05$ using a twolevel hierarchy SW-CRT structure of individuals nested within villages. Estimates of diarrheal prevalence and household size were based on previous pilot work, and included an expected $50 \%$ increase in diarrheal prevalence in all villages during the wet season (June-October). ${ }^{31}$ We used a within-village intracluster correlation coefficient (ICC) of 0.02 (conservative relative to the value of 0.01 obtained from a large clusterrandomized trial in India ${ }^{32}$ ). Based on previous systematic reviews, ${ }^{18,33,34}$ we estimated a $35 \%$ relative reduction in selfreported diarrhea post-intervention. Numerical simulation (with 20,000 simulation replicates) estimated a required sample size of 430 households (with an average household size of five persons) from each of four villages to detect a $35 \%$ reduction from a baseline diarrheal prevalence of $3 \%$, with $80 \%$ power using the regression analysis method described in the Statistical analysis section. We also verified that the type I error rate of $5 \%$ was preserved. To allow for attrition across the five repeat surveys, we approached and recruited all consenting households to ensure we retained adequate power for analysis of the primary outcome.

Statistical analysis. We analyzed participants according to their randomized assignment (intention to treat). The primary outcome (diarrheal prevalence) was calculated for each cell in the stepped wedge design by aggregating over all individuals 
surveyed in each village during each time period. Where zero cases were reported for a given cell, this was replaced with a value of 0.5 cases. Estimation of intervention effects was obtained from a linear regression of the logarithm of the aggregated prevalence, adjusting for seasonal effects and incorporating village as a fixed effect. This is a robust and efficient analysis approach when cluster sizes are large. ${ }^{35}$ Results are presented as the estimated relative reduction (with $95 \% \mathrm{Cl}$ ) in overall prevalence of diarrhea in the intervention periods (riverbank filtration-treated water) compared with control periods (unfiltered river water), obtained by exponentiating the "treatment" regression coefficient from the regression model. Analyses of secondary outcomes were made using the same methods. Analyses were performed in Stata (version 15.0, StataCorp LLC, College Station, TX).

Protocol. We conducted our trial according to our predefined published protocol, ${ }^{24}$ except that heavy monsoonal flooding in northern Karnataka in mid-2016 delayed the construction of water distribution systems, which widened the gap between the start of enrollment and the start of the SW-CRT (start of T1) from 3 to 9 months.

\section{RESULTS}

A flow diagram of enrollment and participation is shown in Figure 2. From 21 villages assessed, four were identified as best meeting eligibility criteria for inclusion. Surveyors identified and enrolled 10,259 participants from 2,304 households across the four villages in the pretrial period (T0). In the pretrial (TO) survey, the main source of drinking water for the majority of households across all four villages $(1,949 / 2,304 ; 85 \%)$ was untreated river water, piped to a standpipe located adjacent to or near the household compound. Most of the remaining households sourced drinking water from a community bore well $(152 / 2,304 ; 7 \%)$ or fetched water directly from the river (145/2,304; 6\%). Following construction of new water distribution systems, 82 households from village 1 were excluded from the study as they were located outside of the intervention delivery zone, reducing the study population to 2,222 households (9,836 participants). Mean cluster size was 531 households (range: 485-583). Losses to follow-up are shown in Figure 2; in total, 331 of 2,222 households (15\%) did not complete all follow-up surveys. Reasons for loss to follow-up were not available for all households; however, where data were available for loss to follow-up, the most common reason was absence from home. Although the overall attrition rate (15\%) was low, the attrition rate within each cluster ranged from $10 \%$ in village 3 to $24 \%$ in village 1 .

Baseline characteristics (T1) of the households that contributed outcome data (2,122 households; 9,433 participants) are presented in Table 1 . The study population consisted of 6,674 adults $(71 \%)$ and 2,759 children (29\%). Household size and individual characteristics were similar across clusters, although household heads in villages 1 and 2 had higher literacy rates than those in the other villages. Most households $(2,058 / 2,122,91 \%)$ owned a mobile phone, and few $(76 / 2,122 ; 4 \%)$ owned a refrigerator. Most households $(1,926 / 2,122 ; 97 \%)$ reported an electricity connection, but electricity supply was intermittent, generally averaging 4-6 hours per day. River water $(1,796 / 2,122 ; 85 \%)$ continued to be the main drinking water source for most households. Most households stored water at home and reported treating stored water; however, the most commonly reported treatment method was straining water through a cloth $(79 \%)$ and few households (14\%) used treatment strategies likely to improve microbiological quality (e.g., filtration, boiling, or addition of chlorine).

Diarrhea prevalence was less than the $3 \%$ anticipated in our sample size calculations in all villages, except village 3 (Table 2). In villages 1, 2, and 4, we noted a marked drop-off in diarrhea reporting between the pretrial (TO) and subsequent surveys, with further reductions over time. By contrast, diarrhea reporting in village 3 continued throughout the study, with an expected seasonal peak during the monsoon season (T4). No significant difference was observed in the timeadjusted relative risk of diarrhea pre- and post-intervention (RR: 0.98 [95\% Cl: 0.24-4.09], $P=0.98$ ) (Table 3). Similarly, we did not observe any significant differences in the timeadjusted relative risk of secondary health outcomes, or negative control symptoms pre- and post-intervention. The final column of Table 3 indicates the possible existence of differential effects of the intervention across villages. Villagespecific relative risks are provided in Supplemental Table 1, and we discuss the potential for these findings to be spurious in the Discussion section.

At each survey, households were asked to estimate the average household daily volume of water use in full buckets of approximately $10 \mathrm{~L}$ capacity (Table 4). The reported average daily volume used during the trial (mean: $234 \mathrm{~L}$; T1-T5 surveys) was not noticeably different from the average daily volume used before intervention construction (mean: $231 \mathrm{~L}$; T0 survey); nor was there a difference in reported water usage during periods in the control condition (mean: $236 \mathrm{~L}$ ) versus the intervention condition (mean: $232 \mathrm{~L}$ ).

Households were asked to report their main source of drinking water and other sources of drinking water at each survey. Notably, households frequently reported drinking water from more than one source (mean: 2.7; SD: 1.1, range: 1-7). Reported uptake of water from the project water storage tanks as a drinking water source changed over time and was highest in the final (T5) survey (Table 5). However, markedly different patterns of uptake were seen between villages, with little uptake until the final survey (T5) in village 1, variable uptake over time in village 3 , relatively consistent uptake in village 4 , and essentially no uptake in village 2 (the last village to receive the intervention). Overall, $31 \%(666 / 2,122)$ of households reported drinking water from the installed water storage tanks during control periods, but only $12 \%$ reported it as their main drinking water source (Table 5). Similarly, 34\% (716/ $2,122)$ reported drinking tank water during periods of intervention delivery, but only $2 \%$ reported it as their main drinking water source.

The hydraulic performance and chemical and bacterial removal efficiencies of our riverbank filtration intervention have been detailed in a previous report. ${ }^{28}$ Briefly, we observed Escherichia coli removal percentages of $>99.9 \%$ (equivalent to three $\log _{10}$ units) during periods when $E$. coli concentrations in the river were highest. ${ }^{28}$ Only one well field (village 3 ) received continuous power from the electrical grid; this well yielded $>100 \mathrm{~L}$ per capita per day. Riverbank filtration treatment performance and well yields in the other three villages were affected by the intermittent availability of electricity; yields in villages 1 and 2 were sometimes insufficient to fill all project tanks. High levels of groundwater salinity in the study area 




FIGURE 2. Flow diagram of sampling, participation, and intervention assignment.

resulted in sodium levels in riverbank filtration-treated water intermittently exceeding the recommended taste threshold.

At the time of site selection, none of the four villages had a community-level safely managed drinking water source. However, between November 2015 and April 2016 (after site selection but before the SW-CRT), reverse osmosis water treatment facilities were built in a central location in each of the four study villages as part of a rural drinking water initiative implemented by the state government. Facilities were operated by Gram Panchayats, with water available at a cost of 2-5 Indian rupees (equivalent to USD\$0.03-\$0.07) per $20 \mathrm{~L}$ collected. Approximately half of study households (1,030/2, 
TABLE 1

Baseline characteristics of villages, households, and participants contributing outcome data (2,122 households)

\begin{tabular}{|c|c|c|c|c|c|}
\hline Item & Village 1 & Village 2 & Village 3 & Village 4 & Total \\
\hline \multicolumn{6}{|l|}{ Village (cluster) characteristics } \\
\hline Number of households, $n$ & 540 & 485 & 583 & 514 & 2,122 \\
\hline Number of residents, $n$ & 2,469 & 1,911 & 2,565 & 2,488 & 9,433 \\
\hline Number of adults ( 18 years and older) & $1,803(73 \%)$ & $1,451(76 \%)$ & $1,696(66 \%)$ & $1,724(69 \%)$ & 6,674 (71\%) \\
\hline Number of children aged $<18$ years & $666(27 \%)$ & $460(24 \%)$ & 869 (34\%) & $764(31 \%)$ & $2,759(29 \%)$ \\
\hline Number of children aged $<5$ years & $154(6 \%)$ & $93(5 \%)$ & $208(8 \%)$ & $141(6 \%)$ & $596(6 \%)$ \\
\hline \multicolumn{6}{|l|}{ Individual characteristics $(n=9,433)$} \\
\hline Age, mean (SD) & $32.4(20.2)$ & $33.5(20.0)$ & $28.5(19.6)$ & $30.5(19.8)$ & $31.1(20.0)$ \\
\hline Male & $1,258(51 \%)$ & $964(50 \%)$ & $1,295(50 \%)$ & $1,237(50 \%)$ & $4,754(50 \%)$ \\
\hline \multicolumn{6}{|l|}{ Household characteristics } \\
\hline Household size, mean (SD) & $4.6(2.1)$ & $3.9(1.6)$ & $4.4(2.0)$ & $4.8(2.4)$ & $4.4(2.1)$ \\
\hline Child aged younger than 5 years & $111(21 \%)$ & 77 (16\%) & $151(26 \%)$ & $105(20 \%)$ & $444(21 \%)$ \\
\hline \multicolumn{6}{|l|}{$\begin{array}{l}\text { Household head characteristics } \\
\quad(n=2,122)\end{array}$} \\
\hline Male & 457 (85\%) & $376(78 \%)$ & $498(85 \%)$ & $392(76 \%)$ & $1,723(81 \%)$ \\
\hline Not literate & $157(32 \%)$ & $185(34 \%)$ & $295(51 \%)$ & $263(51 \%)$ & $900(42 \%)$ \\
\hline \multicolumn{6}{|l|}{ Household asset ownership* } \\
\hline Electricity & $522(97 \%)$ & $473(98 \%)$ & 557 (96\%) & 506 (98\%) & $2,058(97 \%)$ \\
\hline LPG (gas) cooking fuel & $230(43 \%)$ & $355(73 \%)$ & 199 (34\%) & $183(36 \%)$ & $967(46 \%)$ \\
\hline Mobile phone (cell phone) & $493(91 \%)$ & 447 (92\%) & $539(92 \%)$ & $447(87 \%)$ & $1,926(91 \%)$ \\
\hline Television & 408 (76\%) & $373(77 \%)$ & 434 (75\%) & $355(69 \%)$ & $1,570(74 \%)$ \\
\hline Motorbike/scooter & $274(51 \%)$ & $280(58 \%)$ & $271(46 \%)$ & $234(46 \%)$ & $1,059(50 \%)$ \\
\hline Refrigerator & $30(6 \%)$ & $34(7 \%)$ & $1(0 \%)$ & $11(2 \%)$ & $76(4 \%)$ \\
\hline \multicolumn{6}{|l|}{ Household drinking water } \\
\hline River water is the main source & $354(66 \%)$ & 485 (100\%) & $498(86 \%)$ & 459 (89\%) & $1,796(85 \%)$ \\
\hline Bore water is the main source & $185(34 \%)$ & $0(0 \%)$ & $7(1 \%)$ & $0(0 \%)$ & $192(9 \%)$ \\
\hline Store drinking water in the home & 457 (85\%) & 482 (99\%) & 578 (99\%) & $464(90 \%)$ & $1,981(93 \%)$ \\
\hline Treat stored drinking water† & $421(92 \%)$ & 478 (99\%) & $350(61 \%)$ & $357(77 \%)$ & $1,606(81 \%)$ \\
\hline \multicolumn{6}{|l|}{$\begin{array}{l}\text { Sanitation practices: main location of } \\
\text { defecation }\end{array}$} \\
\hline Open defecation & $93(17 \%)$ & $156(32 \%)$ & $434(74 \%)$ & $330(64 \%)$ & $1,013(48 \%)$ \\
\hline Home latrine & $291(54 \%)$ & 235 (49\%) & $143(25 \%)$ & 184 (36\%) & $853(40 \%)$ \\
\hline Shared latrine & $156(29 \%)$ & $94(19 \%)$ & $6(1 \%)$ & $0(0 \%)$ & $256(12 \%)$ \\
\hline \multicolumn{6}{|l|}{ Handwashing station } \\
\hline Located inside & $352(65 \%)$ & 288 (59\%) & $342(59 \%)$ & $229(45 \%)$ & $1,211(57 \%)$ \\
\hline $\begin{array}{l}\text { Soap or detergent observed at } \\
\text { handwashing station } \neq\end{array}$ & $365 / 494(74 \%)$ & $350 / 381(92 \%)$ & $345 / 565(61 \%)$ & $221 / 410(54 \%)$ & $1,281 / 1,850(69 \%)$ \\
\hline
\end{tabular}

Data are $n(\%)$ of households unless otherwise specified.

* Less than $1 \%$ of households reported ownership of a washing machine, computer, air-conditioning, or a dial-up internet connection.

† Data expressed as the proportion of households who reported treating stored water using one or more of the following methods: strain through cloth, let stand and settle, electronic or ceramic filtration, boiling, or addition of chlorine, bleach, or potassium permanganate.

$\ddagger$ Data expressed as the proportion of observed handwashing stations at which soap or detergent was seen.

$122 ; 49 \%$ ) reported using reverse osmosis-treated water as a source of drinking water in at least one of the T1-T5 surveys, although less than half of these $(359 / 2,122 ; 17 \%)$ reported it as their main drinking water source. Reported usage was highest in villages 3 and 4; this finding matched data made available to us by local governments showing that monthly reverse osmosis-treated water facility outputs (liters dispensed) averaged $>10,000 \mathrm{~L}$ per month for villages 1 and 2 and $>100,000 \mathrm{~L}$ per month for villages 3 and 4 . Seasonal variation in reverse osmosis water output was noted, with the highest monthly outputs in all four villages recorded in the drier months of April and May (pre-monsoon).

\section{DISCUSSION}

This trial was designed to assess the impact of communitylevel improvement of water quality and access versus improvement of water access alone on the health of adults and children living in rural Indian villages using a SW-CRT design. We hypothesized that the supply of riverbank filtration-treated water to water storage tanks (intervention) would reduce reported diarrhea rates in adults and children in comparison to the supply of unfiltered water (control). We did not observe a measurable reduction in diarrhea (RR: $0.98[95 \% \mathrm{Cl}$ : 0.24-4.09]) following intervention introduction. During the trial, we observed lower-than-expected reported rates of diarrhea and other health outcomes, and low intervention uptake.

There are several possible explanations for why no reduction in diarrhea attributable to the intervention was observed. First, high intervention uptake is essential to realize health gains from water quality interventions, ${ }^{36}$ and the uptake of our intervention was unexpectedly low. The availability of an alternate, "competitor" safe water supply due to the rollout of reverse osmosis facilities in study villages just before SW-CRT commencement likely affected intervention uptake, and therefore impact evaluation. We did not expect introduction of this competing water supply, given our exclusion criterion of known plans for a water supply upgrade, and its rollout timing was unfortunate. However, a pragmatic reality is that intervening factors can appear during planning and early implementation stages of a trial, and it is not reasonable to expect villages to forego such improvements while awaiting a study intervention. Intervention uptake in villages 1 and 2 may also have been affected by sporadic interruptions in water supply due to intermittent electricity availability. Second, the unexpectedly low prevalence rates of self-reported diarrhea, 
TABLE 2

Diarrhea prevalence ${ }^{*}$ by village and time period

\begin{tabular}{|c|c|c|c|c|c|c|c|c|c|c|}
\hline \multirow[b]{2}{*}{ Time period } & \multicolumn{2}{|c|}{ Village 1} & \multicolumn{2}{|c|}{ Village 3} & \multicolumn{2}{|c|}{ Village 4} & \multicolumn{2}{|c|}{ Village 2} & \multicolumn{2}{|r|}{ Total } \\
\hline & Population (n) & Prevalence (\%) & Population (n) & Prevalence (\%) & Population (n) & Prevalence (\%) & Population (n) & Prevalence (\%) & Population (n) & Mean prevalence (\%) \\
\hline Pretrial (T0) & 2,970 & 0.7 & 2,567 & 1.3 & 2,587 & 2.8 & 2,135 & 1.6 & 10,259 & 1.6 \\
\hline $\mathrm{T} 1$ & 2,469 & $0.0 \dagger$ & 2,565 & 2.9 & 2,488 & 0.6 & 1,911 & $0.0 \dagger$ & 9,433 & 0.9 \\
\hline $\mathrm{T} 2$ & 2,281 & $0.0 \dagger$ & 2,564 & 0.9 & 2,414 & 0.2 & 1,798 & 0.1 & 9,057 & 0.3 \\
\hline T3 & 2,254 & $0.0 \dagger$ & 2,546 & 3.1 & 2,353 & 0.1 & 1,782 & 0.1 & 8,935 & 0.9 \\
\hline $\mathrm{T} 4$ & 2,234 & $0.0 \dagger$ & 2,493 & 4.3 & 2,127 & $0.0 \dagger$ & 1,678 & $0.0 \dagger$ & 8,532 & 1.3 \\
\hline T5 & 2,202 & $0.0 \dagger$ & 2,486 & 3.0 & 2,086 & $0.0 \dagger$ & 1,654 & 0.0† & 8,428 & 0.9 \\
\hline
\end{tabular}

* Seven-day-period prevalence of diarrhea among adults and children.

† Less than 2 cases of diarrhea reported in entire village (cluster) for this period.

overlaid by a drop-off in reported diarrheal symptoms between the pretrial (TO) and subsequent surveys in three of the four villages, reduced our statistical power to detect a health impact attributable to the intervention. One potential explanation for the low diarrhea rates observed is that the uptake of safe drinking water from reverse osmosis facilities before trial commencement resulted in lower baseline diarrhea rates. Alternatively, these findings could indicate reporting fatigue or a reporting bias, ${ }^{16}$ potentially exacerbated by the long delay between the pretrial (TO) survey and the start of the trial (a result of monsoonal flooding); we will explore this further in qualitative work. Third, our intervention was focused on improving access to safe water and did not address other diarrhea transmission pathways. Although there is no doubt that diarrheal pathogens can be transmitted by contaminated drinking water, the relative importance of water as a transmission pathway remains unclear. ${ }^{6}$ A dramatic disparity exists between the results of blinded and unblinded trials of point-ofuse water quality interventions, with unblinded trials often reporting large protective effects and blinded trials typically yielding null effects. ${ }^{6,37}$ Recent studies suggest that levels of fecal contamination on children's hands may be a stronger predictor of diarrheal illness than fecal contamination levels in stored drinking water. ${ }^{38,39}$ Our intervention did not include improvements to sanitation or handwashing facilities, and in light of the ongoing high rates of open defecation, it is likely that the environment remained fecally contaminated and other environmental diarrhea transmission pathways were important. 6

It is useful in cluster-randomized trials to report the magnitude of the observed ICC. Although our analysis method did not require direct calculation of the ICC, we estimated the ICC for diarrhea for each period of the trial and obtained the average, which was 0.025 (range: $0.005-0.048$ across the five periods). However, we caution the reader that because of our suspicions of spuriously low reporting of diarrhea, this figure of 0.025 may be inaccurate and possibly too large. Although our results indicated no effect of the riverbank filtration intervention when averaged over all villages and controlling for time trends, the statistical tests for interaction indicated possible variation in the intervention effects across the four villages, particularly for the diarrhea and respiratory outcomes. Supplemental Table 1 indicates that in village 3, risk for these outcomes increased with the intervention. However, village 3 was the only village with consistent reporting of diarrhea outcomes over time and least likely to be subject to reporting fatigue/bias. The inherent confounding of intervention and time effects in the stepped wedge design will make a village with stable rates over time appear to have an increased risk due to the intervention in situations where other villages have sharply decreased rates over time. Similarly, Supplemental Table 1 indicates a decreased risk of diarrhea in village 4 due to the complete absence of reported events coinciding with the periods in which it received the intervention. As a result, we consider the differential intervention effects for diarrhea and respiratory infections as an artifact of the reporting bias, rather than representing real increased or decreased risks due to the riverbank filtration intervention.

We chose a cluster-randomized rather than an individually randomized design as our intervention was delivered at a community (cluster) level. The SW-CRT design has become increasingly popular in recent years in the evaluation of service or policy interventions delivered at a cluster level. ${ }^{25,27} \mathrm{~A}$ key aspect of the design is that each cluster contributes observations under both control and intervention conditions, enabling within-cluster comparisons. Most previous trials using this design have been performed in advanced economies and health-care settings. ${ }^{27,40}$ Few SW-CRTs have been

TABLE 3

Overall treatment effect by outcome (7-day-period prevalence); $n=9,433$

\begin{tabular}{|c|c|c|c|c|}
\hline Outcome & Mean prevalence ${ }^{\star}(\%)$ & RR $(95 \% \mathrm{Cl}) \dagger$ & $P$-value $\dagger$ & $P$-value $\neq$ \\
\hline Diarrhea & 0.9 & $0.98(0.24-4.09)$ & 0.979 & 0.002 \\
\hline Acute respiratory infection & 1.2 & $0.62(0.13-3.05)$ & 0.524 & 0.017 \\
\hline Skin infection & 0.1 & $2.62(0.48-14.45)$ & 0.239 & 0.608 \\
\hline Any illness§ & 1.2 & $0.70(0.16-3.06)$ & 0.602 & 0.145 \\
\hline Negative control symptoms $\|$ & 1.1 & $0.86(0.20-3.67)$ & 0.818 & 0.077 \\
\hline Fever & 0.7 & $0.86(0.18-4.13)$ & 0.831 & 0.091 \\
\hline
\end{tabular}

* Mean prevalence across all clusters and all time periods in SW-CRT (T1-T5).

† Using a linear regression model where dependent variable $=$ outcome and independent variable $=$ riverbank filtration intervention, adjusted for time period and cluster.

¥ Using a linear regression model where dependent variable = outcome and independent variable = riverbank filtration intervention, adjusted for time period and cluster, with interaction term between treatment and cluster.

$\S$ Seven-day-period prevalence of any of the following outcomes: diarrhea, acute respiratory infection, or skin infection.

|| Seven-day-period prevalence of any of the following: self- or caregiver-reported bruising, abrasion, urinary frequency, or headache (negative control symptoms). 
TABLE 4

Household water usage, mean number of full buckets (SD)*

\begin{tabular}{|c|c|c|c|c|c|}
\hline Survey & Village 1 & Village 3 & Village 4 & Village 2 & All villages \\
\hline Pretrial (TO) & $22.5(10.7)$ & $18.0(13.0)$ & $23.5(10.9)$ & $28.0(10.8)$ & $23.1(11.7)$ \\
\hline $\mathrm{T} 1$ & $23.9(9.4)$ & $19.6(7.9)$ & $18.1(9.2)$ & $24.3(8.7)$ & $21.4(9.2)$ \\
\hline $\mathrm{T} 2$ & $25.1(10.3)$ & $25.2(10.5)$ & $20.4(9.3)$ & $26.3(7.6)$ & $24.2(9.8)$ \\
\hline T3 & 24.3 (10.3) & $19.5(3.7)$ & $18.0(7.4)$ & $32.5(6.1)$ & $23.2(9.0)$ \\
\hline T4 & $26.4(9.7)$ & $19.0(3.4)$ & $21.9(8.3)$ & $29.8(8.5)$ & $23.9(8.7)$ \\
\hline T5 & $26.6(8.7)$ & $18.2(3.7)$ & $23.1(6.2)$ & $31.0(8.5)$ & $24.3(8.4)$ \\
\hline Average across study (T1-T5) & $25.2(9.8)$ & $20.3(7.0)$ & $20.2(8.4)$ & $28.6(8.5)$ & $23.4(9.1)$ \\
\hline
\end{tabular}

Note: 421/12,261 observations missing (3\%); but 417/421 are from pretrial (T0) survey. The cells having bold values indicate post-intervention periods.

* Householders were asked to estimate how much water their household uses each day (for any purpose) in full buckets of approximately $10 \mathrm{~L}$ capacity.

performed in LMICs; these include only two previously published water intervention SW-CRTs from South Africa ${ }^{41}$ and Mexico. ${ }^{42}$ The SW-CRT is a pragmatic study design which has advantages over parallel cluster trials in terms of increased statistical efficiency (due to within-cluster comparisons). The staggered intervention rollout is particularly appealing if simultaneous installation of the intervention at more than one location has logistical and cost implications. However, it has numerous methodological complexities, and reporting guidance has only recently been published as an extension to the CONSORT statement. ${ }^{27}$ We opted for a SW-CRT design, rather than a parallel cluster-randomized trial design with delivery of the intervention to the control arm at the end of the trial, for reasons of statistical power, budget, and funding duration. ${ }^{25}$ Although we observed that the last village to receive the intervention had the highest attrition rate and the lowest rate of intervention uptake, possibly reflective of frustration in having to wait for intervention implementation, this same "disappointment effect" may have been observed in a parallel cluster-randomized trial design among control participants not scheduled to receive the intervention until after the trial. This highlights the need to sustain participant interest and engagement during control periods, an aspect not specifically targeted in our study.

A full evaluation of the hydraulic performance and chemical and bacterial removal efficiencies of our riverbank filtration intervention has been outlined in a previous report. ${ }^{28}$ As expected, we observed an improvement in the microbiological quality of river water following riverbank filtration treatment. ${ }^{28}$ However, well yields and riverbank filtration treatment performance were affected by electricity supply issues, particularly in villages 1 and 2. Groundwater salinity in the study area, likely a result of local agricultural practices including flood irrigation and liberal application of fertilizers, led to intermittently elevated sodium levels in riverbank filtration-treated water; this likely affected palatability and will be explored further in qualitative work.

In addition to those discussed earlier, our study had a number of limitations. Although we attempted to control for measurement bias by including a negative control outcome, and did not observe differential reporting of diarrhea and negative control symptoms, low sensitivity and responder and observer bias remain as limitations of using self- or caregiverreported health outcomes. ${ }^{6,43}$ Despite increasing recognition of the importance of more objective outcome measures in impact evaluation, optimization remains elusive. ${ }^{37}$ Pathogenspecific laboratory outcomes are appealing, but costs and logistics are deterrents, as is potential misclassification bias, given that fecal pathogen detection does not always equate to illness. $^{44,45}$ Incorporation of only a small number of clusters may limit generalizability of our findings. Based on exclusion criteria for selection, villages were not homogeneous with

TABLE 5

Reported household use of water from project water storage tanks, by village*

\begin{tabular}{|c|c|c|c|c|c|}
\hline & Village 1 & Village 3 & Village 4 & Village 2 & All villages \\
\hline \multicolumn{6}{|l|}{ By survey (time period) } \\
\hline $\begin{array}{l}\text { Use as a drinking water source, } \mathrm{T} 1 \\
\text { survey }\end{array}$ & $14 / 540(3 \%)$ & $255 / 583(44 \%)$ & $150 / 514(29 \%)$ & $0 / 485(0 \%)$ & $419 / 2,122(20 \%)$ \\
\hline $\begin{array}{l}\text { Use as a drinking water source, T2 } \\
\text { survey }\end{array}$ & $15 / 505(3 \%)$ & $61 / 582(10 \%)$ & $258 / 496(52 \%)$ & $10 / 449(2 \%)$ & $344 / 2,032(17 \%)$ \\
\hline $\begin{array}{l}\text { Use as a drinking water source, T3 } \\
\text { survey }\end{array}$ & $7 / 499(1 \%)$ & $44 / 578(8 \%)$ & $118 / 480(25 \%)$ & $0 / 445(0 \%)$ & $169 / 2,002(8 \%)$ \\
\hline $\begin{array}{l}\text { Use as a drinking water source, T4 } \\
\text { survey }\end{array}$ & $18 / 493(4 \%)$ & $150 / 566(27 \%)$ & $175 / 435(40 \%)$ & $0 / 420(0 \%)$ & $343 / 1,914(18 \%)$ \\
\hline $\begin{array}{l}\text { Use as a drinking water source, T5 } \\
\text { survey }\end{array}$ & $193 / 486(40 \%)$ & $100 / 564(18 \%)$ & 196/427 (46\%) & $1 / 414(0 \%)$ & $490 / 1,891(26 \%)$ \\
\hline \multicolumn{6}{|c|}{ By condition (control or intervention) across all trial periods (T1-T5) } \\
\hline $\begin{array}{l}\text { Use as drinking water source during } \\
\text { any control period }\end{array}$ & $14 / 540(3 \%)$ & $280 / 588(48 \%)$ & $362 / 514(70 \%)$ & $10 / 485(2 \%)$ & $666 / 2,122(31 \%)$ \\
\hline $\begin{array}{l}\text { Use as main source of drinking water } \\
\text { during any control period }\end{array}$ & $0 / 540(0 \%)$ & 48/583 (8\%) & 199/514 (39\%) & 0/485 (0\%) & $247 / 2,122(12 \%)$ \\
\hline $\begin{array}{l}\text { Use as a drinking water source during } \\
\text { any intervention period }\end{array}$ & $216 / 540(40 \%)$ & $218 / 583(38 \%)$ & $282 / 514(55 \%)$ & $1 / 485(0 \%)$ & $717 / 2,122(34 \%)$ \\
\hline $\begin{array}{l}\text { Use as main source of drinking water } \\
\text { during any intervention period }\end{array}$ & $25 / 540(5 \%)$ & 2/583 (0\%) & $9 / 514(2 \%)$ & $1 / 485(0 \%)$ & $37 / 2,122(2 \%)$ \\
\hline
\end{tabular}

* This table summarizes households' reported use of water from the project tanks as a drinking water source at the T1-T5 surveys. Unless otherwise specified, data presented are the following: number of households reporting use/number of households surveyed (\%). The cells having bold values indicate intervention periods. Low uptake by villages 1 and 2 may in part be explained by interruptions to electricity supply and lower well yields that resulted in insufficient filling of some tanks (intermittently). 
regard to all baseline characteristics, although we adjusted for intervillage variation within the SW-CRT model. Finally, although we used the same water distribution system for the control and intervention periods, and did not overtly inform participants when the switch occurred, differences in water taste and turbidity, and the activities of the intervention delivery team during the changeover period may have alerted participants to their intervention status. Many community-based interventions in the water sanitation sphere suffer from similar difficulties, making blinding impossible and leading to a potential over- or underestimate of intervention effect due to reporting bias. ${ }^{6,46}$

In this study, we were unable to demonstrate a reduction in diarrhea following the delivery of a riverbank filtration-based water intervention to communities in rural India. The reasons for this are multifactorial, and reflective of the real-world conditions in which environmental interventions are introduced. Our intervention, as conceived, aimed to replace the existing main water supply in study villages (untreated river water) with riverbank filtration-treated water. Although we initially planned to retrofit existing village pipework, deficiencies identified in these systems (including defective pipes and incomplete village coverage) prompted us to construct new pipeline and delivery systems for intervention delivery. An unintended consequence of this was that riverbank filtrationtreated water added to, and therefore competed with, other available sources. This is an inherent problem for any sourcebased community-level water intervention trial where the introduced intervention is not the main water supply.

The shift from the "improved water" target of the Millennium Development Goals to the "safely managed water supply" target of the SDGs recognizes the importance of not just water quality, but access and proximity to water supply. There is therefore a need to develop and deliver safe, affordable, and scalable water interventions that can be delivered on premises. As countries move toward on premises delivery of piped water supplies, increasing uniformity of water delivery systems may better enable the evaluation of community-level interventions. Although our study demonstrates the current difficulty of assessing the impact of real-world water supply interventions using a SW-CRT design, we believe that this design may suit budgetary, funding, and logistical constraints inherent in such evaluations in the future.

Received April 5, 2019. Accepted for publication June 3, 2019.

Published online July 1, 2019.

Note: Supplemental appendix and table appears at www.ajtmh.org.

Acknowledgments: We thank S. P. Luby for his input into the study design and insightful comments on a draft of this manuscript. We gratefully acknowledge the help of our field assistant R. K. Vhaval, and the team of surveyors, builders, maintenance workers, and assistants that worked on this project. We also thank our study participants and their leaders for supporting this study.

Financial support: This research was supported by an Australian National Health and Medical Research Council (NHMRC) project grant (1083408). K. L. (1084351) and A. C. C. (1068732) are supported by NHMRC Career Development Fellowships, and S. L. M. by a NHMRC Postgraduate Scholarship (1115196).

Authors' addresses: Sarah L. McGuinness, Joanne O'Toole, Andrew B. Forbes, S. Fiona Barker, Allen C. Cheng, Martha Sinclair, and Karin Leder, School of Public Health and Preventive Medicine, Monash University, Melbourne, Australia, E-mails: sarah.mcguinness@monash. edu, joanne.otoole@monash.edu, andrew.forbes@monash.edu, fiona.barker@monash.edu, allen.cheng@monash.edu, martha. sinclair@monash.edu, and karin.leder@monash.edu. Thomas B. Boving, Department of Geosciences, University of Rhode Island, Kingston, RI, and Department of Civil and Environmental Engineering, University of Rhode Island, Kingston, RI, E-mail: tboving@uri.edu. Kavita Patil, Fraddry D'Souza, Chetan A. Gaonkar, and Asha Giriyan, The Energy and Resources Institute (TERI), Western Regional Centre, Goa, India, E-mails: kavitah@teri.res.in, fraddry.dsouza@teri.res.in, chetan.gaonkar@teri.res.in, and ashag@teri.res.in.

\section{REFERENCES}

1. GBD 2015 Mortality and Causes of Death Collaborators, 2016. Global, regional, and national life expectancy, all-cause mortality, and cause-specific mortality for 249 causes of death, 1980-2015: a systematic analysis for the Global Burden of Disease Study 2015. Lancet 388: 1459-1544.

2. Walker CL, Rudan I, Liu L, Nair H, Theodoratou E, Bhutta ZA, O'Brien KL, Campbell H, Black RE, 2013. Global burden of childhood pneumonia and diarrhoea. Lancet 381: 1405-1416.

3. Pruss-Ustun A et al., 2014. Burden of disease from inadequate water, sanitation and hygiene in low- and middle-income settings: a retrospective analysis of data from 145 countries. Trop Med Int Health 19: 894-905.

4. World Health Organization, United Nations Children's Fund (UNICEF), 2017. Progress on Drinking Water, Sanitation and Hygiene. 2017 Update and SDG Baselines. Geneva, Switzerland: WHO Press.

5. World Health Organization, 2017. Safely Managed Drinking Water-Thematic Reporting on Drinking Water. Geneva, Switzerland: WHO. Licence: CC BY-NC-SA 3.0 IGO.

6. Schmidt WP, Cairncross S, 2009. Household water treatment in poor populations: is there enough evidence for scaling up now? Environ Sci Technol 43: 986-992.

7. Bartram J, Cairncross S, 2010. Hygiene, sanitation, and water: forgotten foundations of health. PLoS Med 7: e1000367.

8. Stelmach RD, Clasen T, 2015. Household water quantity and health: a systematic review. Int J Environ Res Public Health 12: 5954-5974.

9. Bain R et al., 2014. Global assessment of exposure to faecal contamination through drinking water based on a systematic review. Trop Med Int Health 19: 917-927.

10. GBD Diarrhoeal Diseases Collaborators, 2017. Estimates of global, regional, and national morbidity, mortality, and aetiologies of diarrhoeal diseases: a systematic analysis for the Global Burden of Disease study 2015. Lancet Infect Dis 17: 909-948.

11. Sandhu C, Grischek T, Kumar P, Ray C, 2011. Potential for riverbank filtration in India. Clean Techn Environ Pol 13: 295-316.

12. World Bank, 2017. Reducing Inequalities in Water Supply, Sanitation, and Hygiene in the Era of the Sustainable Development Goals: Synthesis Report of the WASH Poverty Diagnostic Initiative. WASH Poverty Diagnostic. Washington, DC: World Bank.

13. Clasen TF, Alexander KT, Sinclair D, Boisson S, Peletz R, Chang $\mathrm{HH}$, Majorin F, Cairncross S, 2015. Interventions to improve water quality for preventing diarrhoea. Cochrane Database Syst Rev 10: CD004794.

14. Arnold BF, Colford JM Jr., 2007. Treating water with chlorine at point-of-use to improve water quality and reduce child diarrhea in developing countries: a systematic review and metaanalysis. Am J Trop Med Hyg 76: 354-364.

15. Wolf $\mathrm{J}$ et al., 2018. Impact of drinking water, sanitation and handwashing with soap on childhood diarrhoeal disease: updated meta-analysis and meta-regression. Trop Med Int Health 23: 508-525.

16. Hunter PR, 2009. Household water treatment in developing countries: comparing different intervention types using metaregression. Environ Sci Technol 43: 8991-8997.

17. Martin NA, Hulland KRS, Dreibelbis R, Sultana F, Winch PJ, 2018. Sustained adoption of water, sanitation and hygiene interventions: systematic review. Trop Med Int Health 23: 122-135.

18. Waddington H, Snilstveit B, 2009. Effectiveness and sustainability of water, sanitation, and hygiene interventions in combating diarrhoea. J Devel Effectiveness 1: 295-335. 
19. Sobsey MD, Stauber CE, Casanova LM, Brown JM, Elliott MA, 2008. Point of use household drinking water filtration: a practical, effective solution for providing sustained access to safe drinking water in the developing world. Environ Sci Technol 42: $4261-4267$.

20. Luby SP et al., 2018. Effects of water quality, sanitation, handwashing, and nutritional interventions on diarrhoea and child growth in rural Bangladesh: a cluster randomised controlled trial. Lancet Glob Health 6: e302-e315.

21. Null $C$ et al., 2018. Effects of water quality, sanitation, handwashing, and nutritional interventions on diarrhoea and child growth in rural Kenya: a cluster-randomised controlled trial. Lancet Glob Health 6: e316-e329.

22. Parvez SM et al., 2018. Achieving optimal technology and behavioral uptake of single and combined interventions of water, sanitation hygiene and nutrition, in an efficacy trial (WASH benefits) in rural Bangladesh. Trials 19: 358.

23. Schubert J, 2002. Hydraulic aspects of riverbank filtration-field studies. J Hydrol 266: 145-161.

24. McGuinness SL, O'Toole JE, Boving TB, Forbes AB, Sinclair M, Gautam SK, Leder K, 2017. Protocol for a cluster randomised stepped wedge trial assessing the impact of a community-level hygiene intervention and a water intervention using riverbank filtration technology on diarrhoeal prevalence in India. BMJ Open 7: e015036.

25. Hemming K, Haines TP, Chilton PJ, Girling AJ, Lilford RJ, 2015. The stepped wedge cluster randomised trial: rationale, design, analysis, and reporting. BMJ 350: h391.

26. McGuinness SL et al., 2020. Barriers and enablers to intervention uptake and health reporting in a water intervention trial in rural India: a qualitative explanatory study. Am J Trop Med Hyg 102: 507-517.

27. Hemming $\mathrm{K}$ et al., 2018. Reporting of stepped wedge cluster randomised trials: extension of the CONSORT 2010 statement with explanation and elaboration. BMJ 363: k1614

28. Boving TB, Patil K, D'Souza F, Barker SF, McGuinness SL, O'Toole J, Sinclair M, Forbes AB, Leder K, 2019. Performance of riverbank filtration under hydrogeologic conditions along the upper Krishna river in southern India. Water 11. https://doi.org/ 10.3390/w11010012.

29. Centre for Affordable Water and Sanitation Technology, CfAWaST, 2011. WASH Education and Training Resources. Available at: https://resources.cawst.org/ (CC BY-SA 4.0). Accessed January 15, 2015.

30. Arnold BF, Ercumen A, Benjamin-Chung J, Colford JM Jr., 2016. Negative controls to detect selection bias and measurement bias in epidemiologic studies. Epidemiology 27: 637-641.

31. Boving T, Choudri BS, Cady P, Patil K, Reddy R, Davis A, 2010. Clean Water by Riverbank Filtration. Final report prepared for World Bank Development Marketplace Program (DM 2007 Grant 2447).

32. Clasen $\mathrm{T}$ et al., 2014. Effectiveness of a rural sanitation programme on diarrhoea, soil-transmitted helminth infection, and child malnutrition in Odisha, India: a cluster-randomised trial. Lancet Glob Health 2: e645-e653.
33. Wolf $\mathrm{J}$ et al., 2014. Assessing the impact of drinking water and sanitation on diarrhoeal disease in low- and middle-income settings: systematic review and meta-regression. Trop Med Int Health 19: 928-942.

34. Fewtrell L, Kaufmann RB, Kay D, Enanoria W, Haller L, Colford JM Jr., 2005. Water, sanitation, and hygiene interventions to reduce diarrhoea in less developed countries: a systematic review and meta-analysis. Lancet Infect Dis 5: 42-52.

35. Matthews JNS, Forbes AB, 2017. Stepped wedge designs: insights from a design of experiments perspective. Stat Med 36: 3772-3790.

36. Brown J, Clasen T, 2012. High adherence is necessary to realize health gains from water quality interventions. PLoS One 7: e36735.

37. Clasen T, Boisson S, 2016. Assessing the health impact of water quality interventions in low-income settings: concerns associated with blinded trials and the need for objective outcomes. Environ Health Perspect 124: 886-889.

38. Mattioli MC, Davis J, Boehm AB, 2015. Hand-to-mouth contacts result in greater ingestion of feces than dietary water consumption in Tanzania: a quantitative fecal exposure assessment model. Environ Sci Technol 49: 1912-1920.

39. Pickering AJ et al., 2018. Fecal indicator bacteria along multiple environmental transmission pathways (water, hands, food, soil, flies) and subsequent child diarrhea in rural Bangladesh. Environ Sci Technol 52: 7928-7936.

40. Beard E et al., 2015. Stepped wedge randomised controlled trials: systematic review of studies published between 2010 and 2014. Trials 16: 353.

41. Bailey IW, Archer L, 2004. The impact of the introduction of treated water on aspects of community health in a rural community in Kwazulu-Natal, South Africa. Water Sci Technol 50: 105-110.

42. Gruber JS, Reygadas F, Arnold BF, Ray I, Nelson K, Colford JM Jr., 2013. A stepped wedge, cluster-randomized trial of a household UV-disinfection and safe storage drinking water intervention in rural Baja California Sur, Mexico. Am J Trop Med Hyg 89: 238-245.

43. Aiemjoy $\mathrm{K}$ et al., 2018. Defining diarrhea: a population-based validation study of caregiver-reported stool consistency in the Amhara region of Ethiopia. Am J Trop Med Hyg 98: 1013-1020.

44. Kotloff KL et al., 2013. Burden and aetiology of diarrhoeal disease in infants and young children in developing countries (the Global Enteric Multicenter Study, GEMS): a prospective, casecontrol study. Lancet 382: 209-222.

45. Ercumen A, Arnold BF, Naser AM, Unicomb L, Colford JM Jr., Luby SP, 2017. Potential sources of bias in the use of Escherichia coli to measure waterborne diarrhoea risk in low-income settings. Trop Med Int Health 22: 2-11.

46. Wood L, Egger M, Gluud LL, Schulz KF, Juni P, Altman DG, Gluud C, Martin RM, Wood AJ, Sterne JA, 2008. Empirical evidence of bias in treatment effect estimates in controlled trials with different interventions and outcomes: meta-epidemiological study. BMJ 336: 601-605. 